\title{
A Prospective Study of Skin Resurfacing to Maximise Cosmesis and Function in Full Thickness Second Degree Burns
}

\author{
Prof. N. Tamilselvan ${ }^{1}$ M.S., Dr. P.V. Dhanapal ${ }^{2}$ M.S., Dr. S.S. Meera ${ }^{3}$ M.S., \\ ${ }^{I}$ (Associate Professor of General Surgery, Government Mohan Kumaramangalam Medical College, \\ Salem, Tamilnadu, India) \\ ${ }^{2,3}$ (Assistant Professor of General Surgery, Government Mohan Kumaramangalam Medical College, \\ Salem, Tamilnadu, India.)
}

\begin{abstract}
Thanks to improved resuscitation and improvised wound care techniques in burns management during the last few decades, because mortality from burns has considerably reduced for these reasons. Many studies have emphasized on advantages of early intervention and skin grafting in managing full thickness second degree and third degree burns. But this involves creation of another raw area at the donor site and also the resultant scar at the burns area will not have admirable cosmetic results. The aim of our study is management of full thickness second degree burns by tangential excision of the dead tissue, but without skin grafting, that will provide both satisfactory cosmesis and successful functional results. Our study explains a surgical treatment in deep second degree burns, where importance is emphasized on early sequential tangential excision of dead tissue and to rely upon the sweat glands and pilo-sebaceous complex that are present in the sub- cutaneous tissue for reepidermalisation of the burns wound. The result of this study encourages maximized cosmesis and function in burns patients.
\end{abstract}

Keywords: Tangential excision, Full thickness second degree burns

\section{Introduction}

Thanks to improved resuscitation and improvised wound care techniques in burns management during the last few decades, because mortality from burns has considerably reduced for these reasons.. Many studies have emphasized on advantages of early intervention and skin grafting in managing full thickness second degree and third degree burns. But this involves creation of another raw area at the donor site and also the resultant scar at the burns area will not have admirable cosmetic results. The aim of our study is management of full thickness second degree burns by tangential excision of the dead tissue, but without skin grafting, that will provide both satisfactory cosmesis and successful functional results. This study explains a surgical treatment in deep second degree burns, where importance is emphasized on early sequential tangential excision of dead tissue and to rely upon the sweat glands and pilo-sebaceous complex that are present in the sub- cutaneous tissue for reepidermalisation of the burns wound

\section{Aims And Objectives}

To maximize cosmesis and function in full thickness second degree burns using a novel technique of early and sequential tangential excision without skin grafting.

\section{Materials And Methods}

Over last one year, 10 patients with full thickness second degree burns of 20 to $40 \%$ were enrolled in our study. There were 5 males with 5 females with age preponderance of 20 to 50 years. The average age was 35 years and average total body surface burn was $30 \%$. All patients were admitted within 24 hours of the injury and all of them received their initial resuscitation according to Parkland formula i.e. $4 \mathrm{ml}$ of Ringer lactate per kilogram body weight per percentage of burns (limited to 50\% in case the burnt area exceeds 50\%) in the initial 24 hours. And then our novel technique was instituted with co-existant great care avoiding infection, dehydration and electrolyte imbalance. Correction of Anaemia and FFP transfusions were also done in conjunction.

III.1. Inclusion Criteria:

1. Male and female with 20 to $40 \%$ deep ( Full thickness) second degree thermal burns

2. Age between 20 and 50 years

III.2. Exclusion Criteria:

1. Persons with bleeding diathesis.

2. Co morbid hepatic, renal pathologies with hypoproteinemia, diabetes and hypertension. 


\section{Novel Technique Instituted:}

The affected areas were initially cleaned with normal saline and covered with sterile bandage. Fasciotomy was done in the limbs on the second day ( 24 to 48 hours), which facilitated down staging of the burns and helped in recovery of the intermediate zones. Empirical anti-bacterial coverage for gram positive and gram negative organisms were initiated which was later changed to appropriate antibiotics according to culture and sensitivity. In full thickness second degree burns, we started tangential excision on day three itself using a Humby's knife and repeated on day 5 and 7, as and when required. Sequential eschar excision done down to fine punctuate bleeding; (areas with pulsatile bleeding from the subcutaneous tissue were grafted if they are large). Extensive care is implicated in excision of wounds on the dorsum of the hands to avoid hypertrophic scarring and scar webbing of inter-digital spaces. Excision is stopped when the viable tissues are reached. Perfect haemostasis is secured using compression with adrenaline soaked pads. Electrocautery is better avoided which may create a number of small new burnt areas. Sequential and effective debridement repeated as and when needed on day 5 and 7 and skin are allowed to reepithelialise. After reepithelialisation, skin resurfacing was done by wrapping the reepithelialised areas using silca gel pads and tight compression garments if scarring becomes noticeable. Early ambulation, Active range of motion, co-ordination and strengthening exercises were instituted as routine.

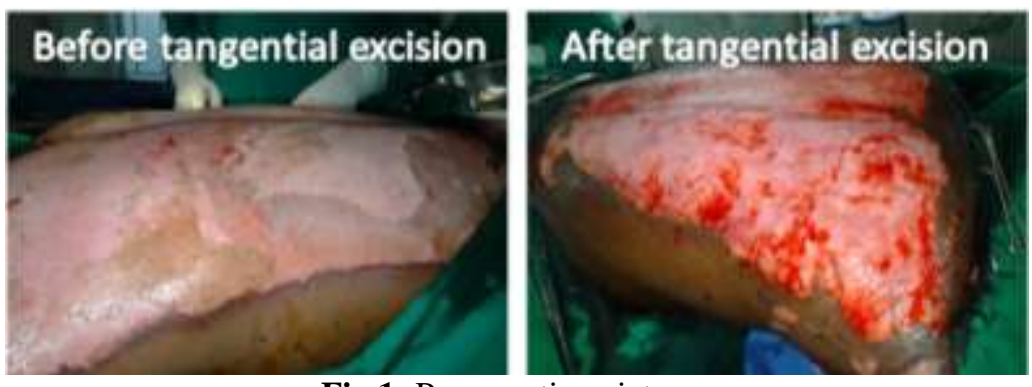

Fig 1: Peroperative picture.
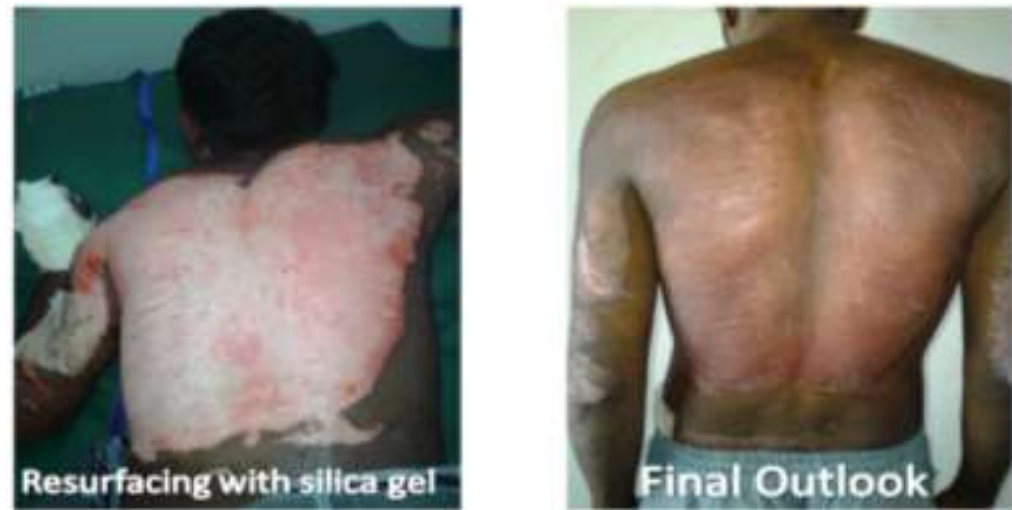

Fig 1: Final outcome
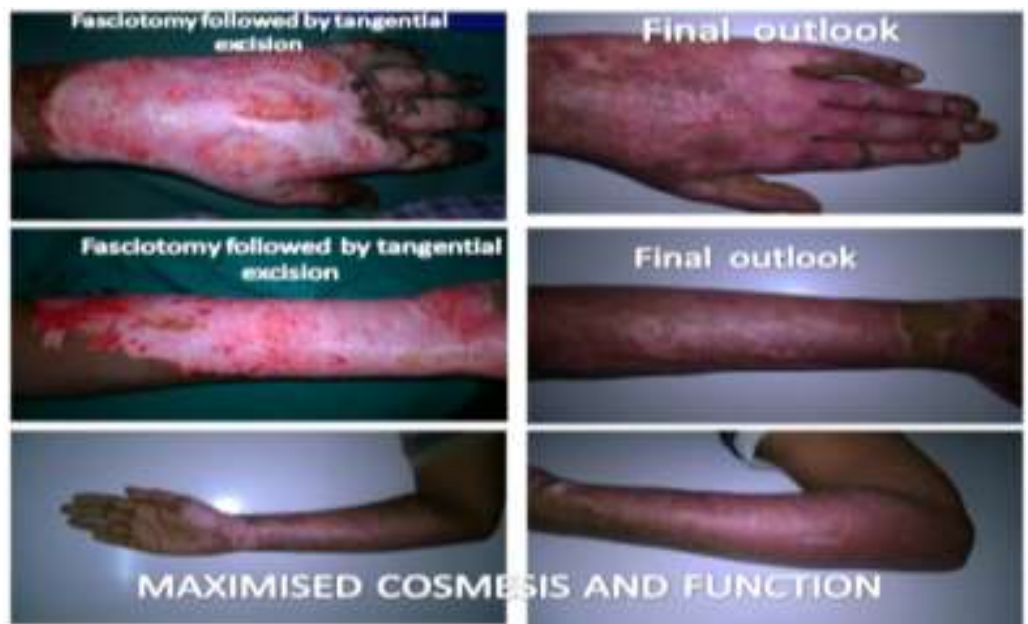

Fig 3: Improved cosmesis and Function. 


\section{Results}

Of the ten cases studied, full range of movements in function zones returned without contractures in all. Minimal scars were observed to be lysed by silica gel under a tight garment. Cosmesis was better than meshed appearance of grafted skin. The result of this study encourages maximized cosmesis and function in face, hands, flexural folds and articular regions.

\section{Discussion}

Though mortality of burns has reduced considerably in last few decades, Burns still constitute a major concern of cosmetic morbidity and functional morbidity to patients. Burns are etiologically classified as thermal burns and electric burns for which the treatment cascade differs. Thermal burns are divided into flame burns and scalds both are managed in same way. First degree Thermal burns usually reepithelialise spontaneously with better cosmetic results within one week to ten days. Superficial second degree burns reepithelialise in two weeks. But the problem of cosmesis and function lies in deep second degree and third degree burns, scarring in the function zones and defaming of the exposed zones. In recent years, tangential excision followed by skin grafting has improvised the results, but post cutaneous graft still impairs the cosmesis with a meshed appearance. Also, the cosmesis and analgesia of donor area is also of concern. So, our study was intended to manage full thickness second degree burns by sequential tangential excision and allowing area to re-epithelialise spontaneously (skin grafting avoided). Cosmesis in exposed zones with minimal scar and mobility in function zones were improved using silica gel pads and compression garments.

Janzekovic first introduced a new concept of early excision and immediate grafting of Burns in 1970 [1]. Lawrence et al studied the same tangential excision technique in detail [2]. Wexler et al [3] and John L. Hunt et al [4] studied the same technique in exposed cosmetic zones such as hands. All the above studies explained the technique of Tangential excision, followed by skin grafting. But our study introduces the idea of no grafting for the first time, with better cosmesis and function results when compared to meshed appearance of grafted areas.

The concept behind this spontaneous reepithelialisation of the raw area after tangential excision is based upon the presence of few functioning sweat glands and pilo-sebaceous complex that are present in the sub-cutaneous tissue and also on the dormant pilo-sebaceous units in the sub-cutaneous tissue. The idea is that, after injury, keratinocytes migrate from all adnexal structures including eccrine and apocrine glands, ducts and pilosebaceous units to the surface and cover the raw area [5]. The dormant pilo-sebaceous units also become active and take part in reepithelialisation. This fact is confirmed by embryological point of view that skin adnexal structures are a group of ectodermally derived appendages that originates as down growths from epidermis to subcutaneous tissue deep to dermis during development [6].

Fasciotomy done in the limbs on the second day ( 24 to 48 hours ) which facilitated down staging of the burns and helped in recovery of the intermediate zones (The zone of stasis) by decreasing the tissue edema. At a time more than $30 \%$ of body surface can be intervened in a single setting by this technique, decreasing the economic burden of the patient's family.

Also by this method, the range of movements of joints returned with $100 \%$ flexibility and patients returned back to their same pre-morbid work without physical and psychological residual trauma. Of the ten cases studied, full range of movements in function zones returned without contractures in all. Minimal scars were observed to be lysed by silica dressing. Cosmesis was better than that of meshed appearance of grafted skin. The result of this study encourages maximized cosmesis and function in both exposed zones (face and hands) and function zones (flexural folds and articular regions).

\section{Conclusion}

By this idea of non-grafting the second degree full thickness burns after tangential excision; we can prevent disfigurement at the donor area, we can get an acceptable cosmetic and functional outcome in burnt area and this method is cost effective.

\section{References}

[1] Janzekovic, Z: A new concept in the early excision and immediate grafting of Burns. J. Trauma, 10:1103,1970

[2] Lawrence, J.C and Carney, S.A: Tangential excision of burns. Br. J. Plastic Surg.,26:93, 1973

[3] Wexler.M.R, Yeschua.R and Neuman.Z, Early treatment of Burns of dorsum of hand by tangential excision and skin grafting. Plast. Reconstr. Surgery 54:268, 1974

[4] John L. Hunt M.D Ronald Sato, M.D. Charles R. Baxter., University of Texas, Early Tangential excision and Immediate mesh auto grafting of deep dermal Hand Burns.

[5] Paul A.J. Kolarsick, BS Maria Ann Kolarsick, MSN, ARNP-C, and Carolyn Goodwin, APRN-BC, FNP, Anatomy and Physiology of Skin.

[6] Development of the skin and its derivatives - Embryology. https://embryology .med. unsw. edu. au/ embryology /.../2015 ANAT2341_Lecture_14_-_Beverdam-Integumentary_Development.pdf 